\title{
Proton bombarded reactions of Calcium target nuclei
}

\author{
Eyyup Tel ${ }^{1}$, Muhittin Sahan ${ }^{1}$, Ismail Hakki Sarpün ${ }^{2}$, Yusuf Kavun ${ }^{3}$, Ali Armagan Gök ${ }^{1}$, Mesut Depedelen ${ }^{1}$ \\ ${ }^{1}$ Osmaniye Korkut Ata University, Department of Physics, Osmaniye, Turkey \\ ${ }^{2}$ Afyon Kocatepe University, Physics Department, Afyonkarahisar, Turkey \\ ${ }^{3}$ Manisa Celal Bayar University, Department of Physics, Manisa, Turkey
}

\begin{abstract}
In this study, proton bombarded nuclear reactions calculations of Calcium target nuclei have been investigated in the incident proton energy range of $1-50 \mathrm{MeV}$. The excitation functions for ${ }^{40} \mathrm{Ca}$ target nuclei reactions have been calculated by using PCROSS nuclear reaction calculation code. Weisskopf-Ewing and the full exciton models were used for equilibrium and for pre-equilibrium calculations, respectively. The excitation functions for ${ }^{40} \mathrm{Ca}$ target nuclei reactions $(\mathrm{p}, \alpha),(\mathrm{p}, \mathrm{n}),(\mathrm{p}, \mathrm{p})$ have been calculated using the semiempirical formula Tel et al. [5].
\end{abstract}

\section{Introduction}

Calcium $(\mathrm{Ca})$ is an important component for the living things and is necessary for human life. Free calcium metal is too reactive to occur in nature and is produced in supernova nucleosynthesis. In supernova explosions, calcium is formed from the reaction of carbon with various numbers of alpha particles (He nuclei), until the most common calcium isotope (containing $10 \mathrm{He}$ nuclei) has been synthesize.

$\mathrm{Ca}$ with atomic number of 20 is an alkaline earth metal and is the most plentiful mineral found in the human body. While the teeth and bones especially contain the most calcium, nerve cells, body tissues, blood, and other body fluids contain the rest of the $\mathrm{Ca}$. $\mathrm{Ca}$ is also an essential trace element in living organisms.

The $\mathrm{Ca}$ has five stable isotopes $\left({ }^{40} \mathrm{Ca},{ }^{42} \mathrm{Ca},{ }^{43} \mathrm{Ca}\right.$, ${ }^{44} \mathrm{Ca}$ and $\left.{ }^{46} \mathrm{Ca}\right)$ and one more $\left({ }^{48} \mathrm{Ca}\right)$ that has such a long half-life. In stellar studies, one of isotopes ${ }^{41} \mathrm{Ca}$ is very important attention due to ${ }^{41} \mathrm{Ca}$ decays to ${ }^{41} \mathrm{~K}$, a critical indicator of solar-system anomalies. The $20 \%$ range in relative mass among naturally occurring calcium isotopes is greater than for any element other than $\mathrm{H}$ and $\mathrm{He}$ [1-3].

\section{Calculation Model and Method}

The PCROSS code uses a unified model based on the solution of the master equation in the form proposed by Cline [6] and Ribansky [7]. Griffin [8] exciton model calculations are used for $n_{0}=1$ (where $n_{0}=1$ is initial exciton number), thus are taken into account direct gamma emission;

$$
n_{e q}=\sqrt{1.4} g E
$$

where $n_{e q}$ is equilibrium exciton number, $g$ is single particle level density, and $E$ is excitation energy of the compound nucleus.

A number of semi-empirical cross section have been proposed for charged and uncharged particles at the different energies. Tel et al. [5] developed the new semi-empirical cross section formulae of reactions induced by proton as follows [6-12];

$$
\sigma_{(p, \alpha)}=C \sigma_{p-n e} e^{a s}
$$

$\sigma_{p-n e}$ is the proton non-elastic cross section and the $C$ and $a$ coefficients are given in Tel et al. [5] for the $(p, \alpha)$ reaction at $17.9 \mathrm{MeV}$ energy. $r_{0}$ is the radius of the nucleus and given as $r_{0}=1.2 \mathrm{fm}$. Coulomb effects for proton induced reactions;

$$
\sigma_{\text {Coul }}=\frac{Z^{2}}{\left(A^{1 / 3}+1\right)}
$$

where $\sigma_{\text {Coul }}$ is the proton Coulomb effect cross section.

Tel et al. [5] suggested that the empirical cross section formulae including proton inelastic cross section [13-16] and Coulomb effects of reactions induced by proton can be expressed as follows,

$$
\sigma_{(p, \alpha)}=C \sigma_{p-n e} \sigma_{C o u l} \exp (a s)
$$

where $\sigma_{p \text {-ne }}$ and $\sigma_{\mathrm{Coul}}$ (in unit of $\mathrm{mb}$ ) are the proton nonelastic and Coulomb effect cross section respectively [17-19]. The coefficients $C$ and $a$ have been determined from only empirical formulae. $\mathrm{Z}$ is the proton number of target nuclei. 


\section{Results and Discussions}

In this study, proton bombarded nuclear reactions calculations of Calcium target nuclei have been investigated in the incident proton energy range of 1$50 \mathrm{MeV}$. The excitation functions for ${ }^{40} \mathrm{Ca}$ target nuclei reactions $(p, \alpha),(p, n),(p, p)$ have been calculated using the semi-empirical formula Tel et al. [5].

The inverse cross sections were obtained by using the optical potential parameters for neutrons proposed by Wilmore et al. [20], for protons proposed by Bechetti et al. [21], and for alpha particles by Huizenga et al. [22]. Full exciton model calculations using with PCROSS code [23] use the initial exciton number as $n_{o}=1$ of 1 neutron and 0 hole. The effects of direct reactions were considered in our calculations.

Theoretical calculations of equilibrium and preequilibrium for ${ }^{40} \mathrm{Ca}(\mathrm{p}, \alpha)$ reactions are seen starting from the same point and showed a similar upward trend, the theoretical equilibrium calculations are just a little higher than pre-equilibrium calculations as seen in Fig. 1. Theoretical calculations of ${ }^{40} \mathrm{Ca}(\mathrm{p}, \mathrm{n})$ show a similar upward trend from around $17 \mathrm{MeV}$ however the theoretical pre-equilibrium calculations are tends to increase after around $20 \mathrm{MeV}$ according to the equilibrium calculations as seen in Fig. 2.

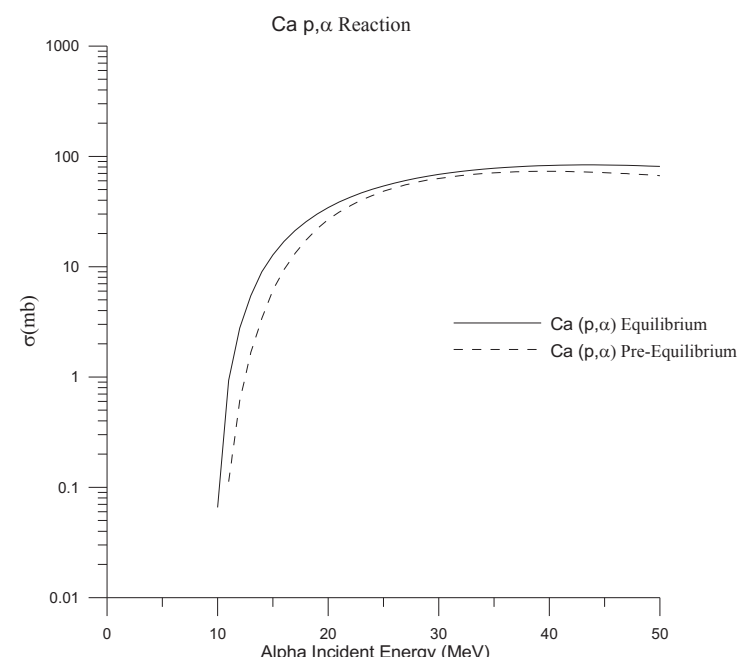

Fig.1. Excitation Function of ${ }^{40} \mathrm{Ca}(\mathrm{p}, \alpha)$ reactions incident proton energy 1-50 MeV.

Theoretical calculations of equilibrium and preequilibrium for ${ }^{40} \mathrm{Ca}(\mathrm{p}, \mathrm{p})$ reaction are seen acquiring around $5 \mathrm{MeV}$ but pre-equilibrium calculations have lower values according to the equilibrium calculations and they have almost the same values and show a similar tendency after $10 \mathrm{MeV}$.

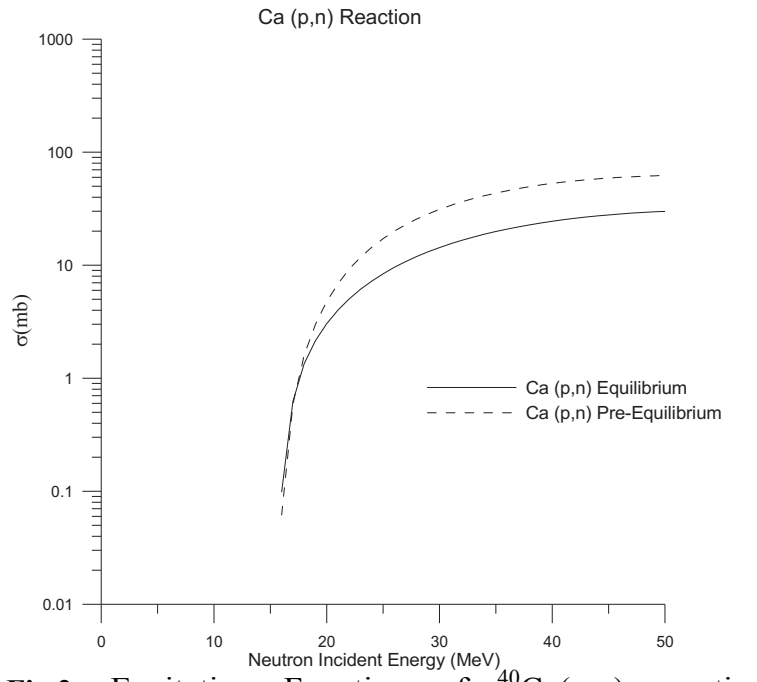

Fig.2. Excitation Function of ${ }^{40} \mathrm{Ca}(\mathrm{p}, \mathrm{n})$ reactions incident proton energy 1-50 MeV.

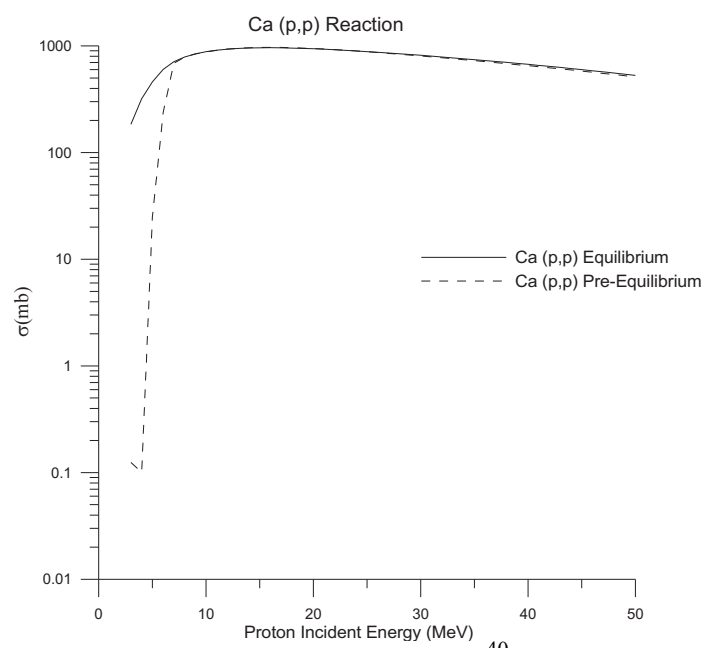

Fig.3. Excitation Function of ${ }^{40} \mathrm{Ca}$ (p,p) reactions incident proton energy 1-50 MeV.

\section{Acknowledgment}

The study was supported by Osmaniye Korkut Ata University (OKU) Science Research Projects Coordination Unit with the grant number: OKÜBAP2017-PT3-023.

\section{References}

1. V.Y. Butko, X. Chi, D.V. Lang, and A.P. Ramirez, Appl. Phys. Lett. https://doi.org/10.1063/1.1631736 83, 4773 (2003)

2. Y.Kavun "Determination of $\mathrm{Sr} / \mathrm{Ca}$ ratios in teeth samples by photoactivation analysis", Ph.D thesis Manisa Celal Bayar University, 103p. (2017)

3. Y. Kavun et al. "Preliminary results of $\mathrm{Sr} / \mathrm{Ca}$ Ratio study of Teeth Samples by Photoactivation Analysis" EPJ Web of Conferences 128, 02001 (2016)

4. E. Tel, H. M. Sahin, A. Kaplan, A. Aydin, T. Altinok, Ann. Nucl. Energy 35 (2) 220, (2007)

5. E. Tel, A. Aydin, E.G. Aydın, A. Kaplan, O. Yavas, I.A. Reyhancan, PRAMANA Indian Academy of Sciences 74, 6, 931 (2010)

6. C. K. Cline, M. Blann: Nucl. Phys. A 172225 (1971)

7. I. Ribansky et al.: Nucl. Phys. A 205 545. (1973)

8. J. J. Griffin, Phys. Rev. Lett. 17478 (1966) 
9. I. Angeli, Table of nuclear root mean square charge radii, IAEANDS-163, Institute of experimental Physics, Kossuth University, H-4001 Debrecen, Pf.105, Hungary, (June 1999)

10. R.J. Harris, W.B. Shuler, M. Eckhause, R.T. Siegel, R.E. Welsh, Phys. Rev. Lett. 20 505. (1968)

11. J.M. Akkermans et al., Phys. Rev. C 22 73. (1980)

12. M. Belgaid, M. Asghar, Appl. Radiat. Isot. 491497 (1998)

13. M. Yigit, E. Tel, Kerntechnik 79 (2014) 488.

14. M. Yigit, E. Tel, Nucl. Eng. Des. 28037 (2014)

15. S. Akca, E. Tel, S. Gungor, Acta Phys. Pol. A 128 (2015)

16. M. Sahan, E. Tel, H. Sahan, A. Kara, A. Aydin, A. Kaplan, I.H. Sarpun, B. Demir, S. Akca, E. Yildiz, J Fusion Energ 34,493 (2015)

17. V.F. Weisskopf, D.H. Ewing, Phys. Rev. 57472 (1940)

18. W. Dilg et al., Nucl. Phys. A 217 269. (1973)

19. F.C. Williams, Nucl. Phys. A 166231 (1971)

20. D. Wilmore, P.E. Hodgson, Nucl. Phys. 55673 (1964)

21. F.D. Becchetti, G.W. Greenlees, Phys. Rev. 1821190 (1969)

22. J.R. Huizenga, G. Igo, Nucl. Phys. 29462 (1962)

23. R. Capote et al., Final Report on Research Contract 5472/RB, INDC(CUB)-004 (Higher Institute of Nuclear Science and Technology, Cuba). Translated by the IAEA on March 1991 (PCROSS program code) (1991) 